\title{
Bericht der Redaktion
}

\section{News from the Editorial Staff}

\section{DDS künftig Open Access ohne Embargo}

Mit großer Freude können wir mitteilen, dass die DDS mit Unterstützung der MaxTraeger-Stiftung ab 2021 als Open-Access-Zeitschrift und somit ohne das bisherige einjährige Embargo online kostenfrei verfügbar erscheinen wird. Wir haben in den vergangenen Jahren bereits sehr positive Erfahrungen mit einzelnen Beiträgen und Beiheften im Open-Access-Format gemacht und hoffen, auf diese Weise noch mehr Leser*innen schneller erreichen und die DDS mit ihren Anliegen noch sichtbarer platzieren zu können.

\section{Verabschiedungen und Begrüßungen von Redaktionsmitgliedern}

Den Redaktionsvorsitz hat seit Anfang Januar Detlef Fickermann inne. Er hat den Staffelstab von Isabell van Ackeren übernommen. Neben ihr sind auch Martin Heinrich und Alexandra Schwarz aus der Redaktion und Sylvia Schütze aus ihrer Funktion als Geschäftsführerin ausgeschieden.

Wir danken den ausgeschiedenen Redaktionsmitgliedern und insbesondere Isabell van Ackeren und Martin Heinrich für ihre langjährige Arbeit in der Redaktion. Martin Heinrich ist Ende 2007 in die Redaktion eingetreten. Anfang 2009 übernahm er dann auch deren Geschäftsführung. Als „dienstältestes“ Redaktionsmitglied trat Isabell van Ackeren schon Ende 2004 der Redaktion bei. Mitte 2012 übernahm sie die Geschäftsführung der Redaktion von Martin Heinrich und - nach Änderung der Geschäftsordnung - Anfang 2016 deren Vorsitz. Martin Heinrich und Isabell van Ackeren haben ganz wesentlich zur erfolgreichen Neuausrichtung der DDS beigetragen. Hierfür danken wir ihnen sehr. Unser besonderer Dank gilt auch Sylvia Schütze, die zunächst ab dem Herbst 2008 als Redaktionsassistentin und dann ab 2016 als Geschäftsführerin mit großem Engagement dazu beigetragen hat, dass die DDS sich in den vergangenen Jahren so gut weiterentwickeln konnte. Wir können uns gar nicht vorstellen, wo wir ohne ihre Unterstützung heute stünden.

Wir freuen uns, Prof. Dr. Julia Gerick, Prof. Dr. Kathrin Racherbäumer und JuniorProfessorin Dr. Verena Schreiber als neue Redaktionsmitglieder sowie Dr. Monika Palowski-Göpfert als neue Geschäftsführerin begrüßen zu können. 
Julia Gerick ist seit 2020 Professorin für Schulpädagogik mit dem Schwerpunkt Schulentwicklungsforschung an der TU Braunschweig. Von 2012 bis 2016 war sie als Wissenschaftliche Mitarbeiterin am Institut für Schulentwicklungsforschung an der Technischen Universität Dortmund tätig; dort hatte sie die Nationale Projektleitung der International Computer and Information Literacy Study (IEA-ICILS 2013) inne. 2013 promovierte sie an der Universität Hamburg zum Thema „Führung und Gesundheit in der Organisation Schule“. Von 2016 bis 2020 war sie Juniorprofessorin für Erziehungswissenschaft unter besonderer Berücksichtigung der Schulpädagogik mit dem Schwerpunkt Schulentwicklungsforschung an der Universität Hamburg. Die Arbeits- und Forschungsschwerpunkte von Julia Gerick sind Schulentwicklungsforschung, Schulqualität, Digitale Medien in Schule und Unterricht, Lehrer*innengesundheit, Schulleitung und Schulleistungsstudien.

Kathrin Racherbäumer ist seit 2019 Professorin für Erziehungswissenschaft mit dem Schwerpunkt Schul- und Unterrichtsentwicklung in der Sekundarstufe an der Universität Siegen. Nach ihrem Studium der Pädagogik (Diplom) an der Universität Duisburg-Essen war sie von 2005 bis 2011 Mitarbeiterin am Zentrum für Lehrerbildung sowie 2006 bis 2009 Wissenschaftliche Mitarbeiterin an der Fakultät Bildungswissenschaften der Universität Duisburg-Essen, zunächst in der Projektkoordination von „indive“ und seit 2009 als Leiterin dieses Projekts. Sie promovierte an der Universität Duisburg-Essen zum Thema „Hochbegabte Schulanfänger und Schulanfängerinnen“. Nach der Leitung weiterer Projekte, u.a. zu „Strategien der Qualitätsentwicklung von Schulen in schwieriger Lage“ und $\mathrm{zu}$ "Sprachsensibler Schulentwicklung“, vertrat sie von 2016 bis 2018 die Professur „Inklusive Pädagogik und Diversität“ am Institut für Pädagogik der Universität Duisburg-Essen und von 2018 bis 2019 die Professur Schulforschung mit dem Schwerpunkt Unterrichtsentwicklung an der Universität zu Köln. Die Arbeits- und Forschungsschwerpunkte von Kathrin Racherbäumer sind Rekonstruktive Schul- und Unterrichts(entwicklungs)forschung im Kontext von Bildungsgerechtigkeit, Inklusion und Heterogenität, Kulturelle Bildung im Kontext von Inklusion und Schulentwicklung, Professionsforschung, Übergänge im Bildungssystem und (Hoch-)Begabungsforschung.

Verena Schreiber ist seit Oktober 2015 als Junior-Professorin für Geographie und ihre Didaktik mit dem Schwerpunkt Humangeographie an der Pädagogischen Hochschule Freiburg tätig. Nach ihrem Studium der Geographie, Katholischen Theologie und Deutschen Philologie an der Johannes Gutenberg-Universität Mainz war sie zunächst Wissenschaftliche Mitarbeiterin an den Universitäten Mainz und Osnabrück, bevor sie 2005 an die Goethe-Universität Frankfurt am Main wechselte. Dort promovierte sie 2010 zum Thema "Städtische Sicherheitsproduktion“. Vor ihrem Wechsel nach Freiburg forschte sie von März bis Juli 2015 am Department of Geography der Loughborough University (UK) zum Themenfeld „Geographies of Childhood, Families and Education“. Verena Schreiber vertritt eine gesellschaftstheoretisch-in- 
formierte sowie empirisch-orientierte Forschung in der Geographiedidaktik. Ihre bildungsgeographische Forschung nimmt insbesondere strukturelle, sozialräumliche und situative Lernvoraussetzungen in den Blick und geht der Frage nach, unter welchen Bedingungen Kinder und junge Menschen gegenwärtig aufwachsen und wie jene auf Bildungsprozesse Einfluss nehmen.

Monika Palowski-Göpfert ist seit 2010 Wissenschaftliche Mitarbeiterin der Wissenschaftlichen Einrichtung Oberstufen-Kolleg (WE_OS) an der Fakultät für Erziehungswissenschaft der Universität Bielefeld. Sie studierte Soziologie an der Universität Bielefeld. Im Kontext ihrer wissenschaftlichen Mitarbeit begleitete bzw. leitete sie mehrere Forschungs- und Entwicklungsprojekte am Oberstufen-Kolleg; ihre Schwerpunkte waren dabei insbesondere riskante Bildungsbiografien in der Sekundarstufe II und Gesundheit bzw. Belastungsempfinden von Lehrkräften, aber auch übergeordnete Fragen von Praxisforschung und Transfer. Gegenstand ihrer 2015 erschienenen Dissertation ist eine Wissenssoziologische Diskursanalyse zur Klassenwiederholung in akademischen und printmedialen Diskursen. Zwischen 2016 und 2019 war sie außerdem in ein internationales Exkursionsprogramm für Bielefelder Lehramtsstudierende innerhalb des Projektes BiProfessional (Qualitätsoffensive Lehrerbildung) eingebunden. $\mathrm{Zu}$ ihren Interessen zählen u.a. Fragen der Bildungsungleichheit und der Schulkultur sowie qualitative und Mixed-Methods-Forschungsansätze.

https://doi.org/10.31244/dds.2021.01.01 